\title{
BMJ Open Adapting the UCLA 3-item loneliness scale for community-based depressive symptoms screening interview among older Chinese: a cross-sectional study
}

\author{
Tianyin Liu (D) , Shiyu Lu, Dara K Y Leung, Lesley C Y Sze, Wai Wai Kwok, \\ Jennifer Y M Tang, Hao Luo (1) , Terry Y S Lum, Gloria H Y Wong (D)
}

To cite: Liu T, Lu S, Leung DKY, et al. Adapting the UCLA 3-item loneliness scale for community-based depressive symptoms screening interview among older Chinese: a crosssectional study. BMJ Open 2020;10:e041921. doi:10.1136/ bmjopen-2020-041921

- Prepublication history and additional materials for this paper is available online. To view these files, please visit the journal online (http://dx.doi. org/10.1136/bmjopen-2020041921).

Received 20 June 2020 Revised 24 November 2020 Accepted 25 November 2020

Check for updates

(C) Author(s) (or their employer(s)) 2020. Re-use permitted under CC BY-NC. No commercial re-use. See rights and permissions. Published by BMJ.

Department of Social Work and Social Administration, The University of Hong Kong, Hong Kong SAR, China

Correspondence to

Dr Gloria H Y Wong;

ghywong@hku.hk

\section{ABSTRACT}

Objective Loneliness is a significant and independent risk factor for depression in later life. Particularly in Asian culture, older people may find it less stigmatising to express Ioneliness than depression. This study aimed to adapt a simple loneliness screen for use in older Chinese, and to ascertain its relevance in detecting depressive symptoms as a community screening tool.

Design, setting and participants This cross-sectional study was conducted among 1653 older adults aged 60 years or above living in the community in Hong Kong. This was a convenient sample recruited from four local non-governmental organisations providing community eldercare or mental healthcare services. All data was collected by trained social workers through face-to-face interviews.

Measures Loneliness was measured using an adapted Chinese version of UCLA 3-item Loneliness Scale, depression symptoms were assessed using the Patient Health Questionnaire-9 (PHQ-9), and social support with emotional and instrumental support proxies (number of people who can offer help). Basic demographics including age, gender, education and living arrangement were also recorded.

Results The average loneliness score was $3.9 \pm 3.0$, and it had a moderate correlation with depressive symptoms $(r=0.41, p<0.01)$. A loneliness score of 3 can distinguish those without depression from those with mild or more significant depressive symptoms, defined as a PHQ9 score of $\geq 5$ (sensitivity $76 \%$, specificity $62 \%$, area under the curve $=0.73 \pm 0.01$ ). Loneliness explained $18 \%$ unique variance of depressive symptoms, adding to age, living arrangement and emotional support as significant predictors.

Conclusion A 3-item loneliness scale can reasonably identify older Chinese who are experiencing depressive symptoms as a quick community screening tool. Its wider use may facilitate early detection of depression, especially in cultures with strong mental health stigma.

Trial registration number ClinicalTrials.gov NCT03593889

\section{INTRODUCTION}

Loneliness is a common human experience. Distinct from objective social isolation,
Strengths and limitations of this study

- This study is the first attempt to use receiver operating characteristic curve analyses to explore how Ioneliness scale may be a screening tool to detect depressive symptoms.

- The recruitment and assessments were done through real-world aged care and mental healthcare services, suggesting feasibility of applying the screening tool in routine services to address the sizeable problem of loneliness and depression in Chinese older adults.

- The participants were not randomly selected, hence it was not a representative sample of communitydwelling older adults in Hong Kong.

- Due to the cross-sectional nature of this study, the findings can only indicate associations between loneliness and depressive symptoms in older adults.

loneliness reflects the individual's experienced dissatisfaction with one's relationships, and it is a distressing feeling that arises from self-assessed discrepancy between actual and desired companionship and/or emotional support. $^{12}$ Because of its subjective nature, loneliness is inevitably affected by the cultural context, ${ }^{3}$ and some of the objective risk factors of isolation, for example, living alone, are not necessarily associated with loneliness. ${ }^{4}$ For instance, cross-cultural research of loneliness in Europe found that older adults in Eastern Europe had higher loneliness score than those in Western Europe, despite that co-residence is more common in Eastern Europe; and this phenomenon was explained by the interplay of individual and societal factors, for example, filial norms are weaker in Western Europe and hence lower co-residence rate fits the norm and does not contribute to higher loneliness. ${ }^{5}$

In eastern cultures, such as Chinese culture that traditionally emphasises family and social cohesion, ${ }^{6}$ loneliness may be more profound 
in situations where family support and social network are weak. A systematic review on loneliness and social support in older Chinese has identified family as the most important source of social support, followed by friends. ${ }^{7}$ Family structure and cohesiveness is rapidly changing in Chinese communities, with increasing number of older persons living alone. ${ }^{6}$ While living alone can be a separate construct from social isolation and loneliness, ${ }^{4}$ empirical studies among Chinese older adults suggested that living alone is associated with stronger feeling of loneliness and higher risk for developing depressive symptoms. ${ }^{8}$ In addition, living alone has more of a negative stigma in Chinese communities than in Western communities, ${ }^{9}$ and is associated with a lower level of perceived social support. ${ }^{10}$ These traditional values and filial expectation are still being held by older persons while more of them are living alone, loneliness is thus expected to be highly prevalent in this population. In earlier cohorts (between 2002 and 2008) of a nationally representative sample of older adults in China, about $28 \%$ reported feeling lonely. ${ }^{11}$

Loneliness is a known risk factor for depression, independent of other demographic and risk factors. ${ }^{12}$ Depression is a common mental health problem in older persons and it poses great burden on healthcare and social care systems, either directly due to the disability it causes or indirectly by complicating other physical health problems and care. ${ }^{13}$ Prevention and early intervention are effective in reducing suffering and societal costs from major depressive disorder; ${ }^{14}$ however, it is often under-recognised and undertreated. ${ }^{15}$ The fact that later-life depression is under-recognised and undertreated may be attributed to stigma and low awareness. In the USA, for example, older adults were generally worse than younger persons at identifying depressive symptoms, and it was suggested that greater efforts are needed in educating them about the symptoms. ${ }^{16}$ This challenge is particularly prominent in Asian cultures, where stigma towards mental illness may be more severe because it is attached to the family as a whole. ${ }^{17}$ In developing countries in Asia, social disapproval and devaluation of families with mentally ill individuals are important barriers to help-seeking. ${ }^{18}$ The more severe and integrated stigma to mental illness can help understand lower reported prevalence rates in these populations. For example, an earlier meta-analysis revealed a much lower prevalence of depression among elders in China, at only $3.86 \%$ compared with $12 \%$ in Western Europe, ${ }^{19}$ and this low prevalence rate may be partly attributed to strong stigma of mental illness. ${ }^{20}$

Loneliness and depression are also conceptually related, although researchers have also taken care to distinguish between the two concepts. For older persons, the word lonely was highly salient when asked to free-list words describing a depressed person or themselves when depressed; they also saw loneliness as less stigmatising than depression, and recognised loneliness as a gateway to depression if left unattended. ${ }^{21}$ In Chinese, the common translation of loneliness is jimo (寂寞), which has meanings of silent, still and desolate; these concepts are distinct from those of depres-

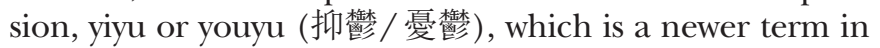

Chinese combining character that have connotations of repression, sadness and melancholy. ${ }^{22}$ Depression and loneliness are highly associated, particularly in older adults, ${ }^{23-25}$ with a magnitude of association ranging between 0.40 and $0.65 .^{1226}$

In view of the role of loneliness in depression among older people, the screening of loneliness is important for detecting and preventing depression. Commonly used validated scales include: the 20-item Revised UCLA Loneliness Scale (R-UCLA); ${ }^{27}$ the 6-item De Jong Gierveld Loneliness Scale; ${ }^{28}{ }^{29}$ and the UCLA 3-item Loneliness short scale (UCLA 3-item). ${ }^{30}$ Among these, the UCLA 3-item scale has gained popularity in large scale surveys, for instance, the Health and Retirement Study ${ }^{31-33}$ and the English Longitudinal Study of Ageing, ${ }^{34}$ as it is sufficiently sensitive to detect loneliness, simple to administer and also less prone to response bias than a single question. ${ }^{33}$ UCLA 3-item assesses subjective feelings of loneliness through questions that avoid using the word 'lonely' or 'loneliness,' but asks about the frequency of feeling. To our knowledge, the potential of using a loneliness screen to detect depressive symptoms in older population is unexplored, and this study aims to examine the utility of a culturally adapted UCLA 3-item scale in detecting depressive symptoms.

\section{METHODS}

\section{Sampling and procedures}

Data used in this study came from a preventive care for community-dwelling older adults at risk of or with subclinical depressive symptoms living in Hong Kong. The inclusion criteria were: (1) aged 60 years or above; (2) living in the service catchment area; and (3) having mild or above depressive symptoms (defined as a score of $>4$ on the Patient Health Questionnaire-9 (PHQ-9) $)^{30}$ and/or having at least one risk factor of depression in old age, including report of loneliness, lack of social interaction, lack of meaningful/enjoyable activities, chronic pain, more than four common chronic diseases and/or recent bereavement. The exclusion criteria were: (1) a known history of autism, intellectual disability, schizophrenia spectrum disorder, bipolar disorder, Parkinson's disease or dementia; and/or (2) significant suicidal risk. Eligible older persons were asked to give consent for participation in the service and the research study. The current analysis used screening data collected from 1807 communitydwelling elders for entry into the service between October 2017 and January 2019. All participants were recruited from four non-governmental organisations providing community eldercare or mental healthcare services by trained social workers through face-to-face interviews.

\section{Instruments and measures}

The locally adapted UCLA 3-item loneliness scale

Participants' loneliness was measured using a locally adapted version of UCLA 3-item. ${ }^{30}$ As UCLA 3-item was originally developed in the West and in English, both the cultural context and language, are different from 
the norms in Chinese. Therefore, cross-cultural adaptation is needed. ${ }^{35}$ For example, in a Japanese version of the UCLA 3-item scale, one of the items required using a passive voice. ${ }^{36}$ Although there were existing Chinese versions, ${ }^{37-39}$ for this study, a spoken Chinese (Cantonese) version is required for the interview, given that a substantial proportion of older persons in Hong Kong had no formal education and cannot read, and the spoken language has terms and usages that differ from the written form. We adopted a five-stage process to ensure that the Cantonese version is a faithful adaptation (see online supplemental table 1 for details). The three items are: in the past 2 weeks, how often do you feel (i) lack of companionship, (ii) left out and (iii) isolated. The 3-point response scale for each item ranges from 'hardly ever or never' ( 1 point) to 'often' (3 points), and the total score is the sum of all items, which ranges from 3 to 9 , with higher scores indicating a higher level of perceived loneliness. We adopted the 4-point Likert scale that was used in the R-UCLA ${ }^{18}$ from which UCLA 3-item was derived.

\section{The Patient Health Questionnaire-9}

Depressive symptoms were assessed using the PHQ-9, ${ }^{40}$ a 9-item instrument that incorporates depression diagnostic criteria, and rates symptom frequency of each factor into the scoring severity index. A PHQ-9 score of 0 to 4 signals no depression, 5 to 9,10 to 14,15 and above indicate mild, moderate, and moderately severe depressive symptoms, respectively. ${ }^{40}$

\section{Social support questions}

Social support is a multidimensional construct. In this study, we focussed on emotional and instrumental support, following previous literature on their roles in social support among older Chinese. ${ }^{41}$ We did not include the subjective evaluation as it overlaps with the concept of loneliness. We used a name generation method following the exchange approach, which has been used in community-dwelling older persons in Hong Kong to understand the role of social support in ageingin-place preference ${ }^{42}$ and depressive symptoms. ${ }^{43}$ We first assessed the presence of helping figure, and then used the number of helpers as proxies for emotional and instrumental support. The questions were 'Do you have someone to talk to when you feel down?' and 'Do you have someone to help you with trivial things (eg, change the bulb)?' If they answered yes, we then asked them to list out the people who offer them help with a maximum number of five people.

\section{The Montreal Cognitive Assessment 5-minute protocol}

Cognition was measured using the Montreal Cognitive Assessment 5-minute protocol (MoCA 5-min) ${ }^{44}$ to screen out probable cognitive impairment. Participants who scored below the second percentile by age and education on MoCA 5-min were excluded from later analyses to avoid potential insight problems. ${ }^{45} 46$
General information questionnaire

We used a self-designed questionnaire to collect demographic information, including age, gender, education (years and highest level attained), work years and living arrangement (living alone versus otherwise).

\section{Statistical methods}

Confirmatory factor analysis (CFA) was used to examine whether the data fitted the theoretically hypothesised one-factor model of UCLA 3-item. Cronbach's alpha estimates and Spearman's item-total correlations were performed to examine the internal consistency and reliability of the scale. Bivariate correlation analyses were used to test the convergent and discriminant validity of the scale with conceptually related variables, that is, depressive symptoms and social support. Multiple regressions with PHQ-9 score as the dependent variable were performed to demonstrate the incremental validity of loneliness in explaining depressive symptoms after controlling for other risk/protective factors, including demographics (female gender and living alone) and social support (emotional and instrumental support). Finally, we used receiver operating characteristic (ROC) curve analyses to identify the cut-off points of loneliness scores in detecting: (a) mild and more serious depressive symptoms using a PHQ-9 score of 5 as gold standard; (b) moderate and more serious depressive symptoms using a PHQ-9 score of 10 as the cut-off; and (c) moderately severe and more serious depressive symptoms using a PHQ-9 score of 15 . The Youden Index ${ }^{47}$ was used to define the optimal cut-offs, a method that has been frequently unitised in biomedical diagnostic practices. The Youden Index $(\mathrm{J})=$ Sensitivity + Specificity -1 ; thus by maximising J across various cut-offs, the optimal cut-off point is calculated. CFA was undertaken using the lavaan package in $\mathrm{R}$ for latent variable analysis, ${ }^{48}$ and all other analyses were conducted using SPSS V.25.0 (IBM Corporation, Armonk, New York, USA).

\section{Patient and public involvement}

Patients and public (older adults with or without depression) advised the cultural adaptation of the UCLA 3-item scale (see online supplemental table 1). Patients and the public were not involved in reporting of the present research.

\section{RESULTS}

\section{Demographic characteristics}

Participants' sociodemographic and clinical characteristics are reported in table 1 . The mean age of participants was $77.98(\mathrm{SD}=8.24)$ years. This cohort had low education, $35 \%$ had no formal education, and average years of schooling was $4.86(\mathrm{SD}=4.40)$. Nearly half of them lived alone $(\mathrm{n}=742,44.9 \%)$ (table 1$)$.

\section{Correlations between loneliness, depression and social support}

The mean total score of the Chinese UCLA 3-item 3.91 $(\mathrm{SD}=3.02)$ with the possible score ranging from 0 to 9 , 


\begin{tabular}{|c|c|c|c|}
\hline Demographics & Category & $\mathbf{N}(\%)$ & Mean (SD) \\
\hline Age, years & & - & 77.98 (8.24) \\
\hline \multirow[t]{2}{*}{ Gender } & Female & $1289(78)$ & - \\
\hline & Male & $364(22)$ & - \\
\hline Work, years & & - & 30.99 (14.78) \\
\hline \multirow[t]{4}{*}{$\begin{array}{l}\text { Education, level } \\
\text { (missing, } n=21 \text { ) }\end{array}$} & 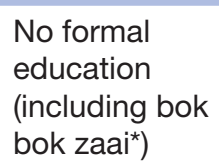 & 575 (34.8) & - \\
\hline & $\begin{array}{l}\text { Primary } \\
\text { education }\end{array}$ & $613(37.1)$ & - \\
\hline & $\begin{array}{l}\text { Secondary } \\
\text { education }\end{array}$ & $376(22.8)$ & - \\
\hline & $\begin{array}{l}\text { Higher } \\
\text { education } \\
\text { (associate } \\
\text { degree or } \\
\text { above) }\end{array}$ & $68(4.1)$ & - \\
\hline
\end{tabular}

\begin{tabular}{|c|c|c|c|}
\hline $\begin{array}{l}\text { Education, } \\
\text { years }\end{array}$ & & - & $4.86(4.40)$ \\
\hline \multirow{2}{*}{$\begin{array}{l}\text { Living } \\
\text { arrangement }\end{array}$} & Alone & 742 (44.9) & - \\
\hline & Otherwise & $911(55.1)$ & - \\
\hline \multirow{7}{*}{$\begin{array}{l}\text { Emotional } \\
\text { support }\end{array}$} & 0 person & $569(34.4)$ & - \\
\hline & 1 person & 765 (46.3) & - \\
\hline & 2 persons & $217(13.1)$ & - \\
\hline & 3 persons & $66(4)$ & - \\
\hline & 4 persons & $23(1.4)$ & - \\
\hline & 5 persons & $13(0.8)$ & - \\
\hline & Total number & - & $0.94(0.94)$ \\
\hline \multirow{7}{*}{$\begin{array}{l}\text { Instrumental } \\
\text { support }\end{array}$} & 0 person & $457(27.7)$ & - \\
\hline & 1 person & $911(55.1)$ & - \\
\hline & 2 persons & $194(11.7)$ & - \\
\hline & 3 persons & $58(3.5)$ & - \\
\hline & 4 persons & $21(1.3)$ & - \\
\hline & 5 persons & $12(0.7)$ & - \\
\hline & Total number & - & $0.98(0.87)$ \\
\hline MoCA 5-min & $\begin{array}{l}\text { Possible score } \\
\text { range ( } 0 \text { to } \\
30) \text {; actual } \\
\text { score range } \\
(9.5 \text { to } 30)\end{array}$ & & $21.05(4.57)$ \\
\hline \multirow[t]{3}{*}{ PHQ-9 } & $\begin{array}{l}\text { Possible score } \\
\text { range (0 to } \\
27) \text {; actual } \\
\text { score range (0 } \\
\text { to } 23 \text { ) }\end{array}$ & - & $6.62(4.13)$ \\
\hline & $\begin{array}{l}0 \text { to } 4 \text { No } \\
\text { depression }\end{array}$ & 468 (28.3) & \\
\hline & $\begin{array}{l}5 \text { to } 9 \text { Mild } \\
\text { depressive } \\
\text { symptoms }\end{array}$ & 874 (52.9) & \\
\hline
\end{tabular}

Continued

\begin{tabular}{|c|c|c|c|}
\hline Demographics & Category & $\mathbf{N}(\%)$ & Mean (SD) \\
\hline & $\begin{array}{l}10 \text { to } 14 \\
\text { Moderate } \\
\text { depressive } \\
\text { symptoms }\end{array}$ & $239(14.5)$ & \\
\hline & $\begin{array}{l}\geq 15 \\
\text { Moderately } \\
\text { severe and } \\
\text { more serious }\end{array}$ & $72(4.4)$ & \\
\hline UCLA 3-item & $\begin{array}{l}\text { Possible score } \\
\text { range (0 to } 9 \text { ); } \\
\text { actual score } \\
\text { range (0 to } 9 \text { ) }\end{array}$ & - & 3.91 (3.02) \\
\hline
\end{tabular}

*bok bok zaai: 1 to 3 years' informal education provided by private institutions in Cantonese speaking regions, gradually disappearing since the New Culture Movement in China in the 1910s. MoCA 5-min, Montreal Cognitive Assessment 5-minute protocol; PHQ-9, Patient Health Questionnaire-9.

and the frequency and percentage of response categories of individual items are summarised in online supplemental table 2. In the CFA results, all factor loadings exceeded 0.80, and the goodness-of-fit indexes, including comparative fit index (CFI), Tucker Lewis index (TLI), root-mean-square error of approximation (RMSEA), and standardised root-mean-square residual (SRMR), showed that the model had very good fit $(\mathrm{CFI}=1.0$; TLI=1.0; RMSEA=0.00; SRMR=0.00), which confirmed the structural validity of the translated scale. ${ }^{49} \mathrm{CFA}$ and the item-total correlations were all above $0.70(\mathrm{p}<0.01)$. The Cronbach's alpha coefficient for the scale was 0.87 , indicating that it has good internal consistency. ${ }^{49}$

The mean PHQ-9 score was $6.62(\mathrm{SD}=4.13)$, according to the validated cut-off scores ${ }^{40} 468(28.3 \%)$ of the participants had no depressive symptoms, nearly half $(\mathrm{n}=874$, $52.9 \%$ ) had mild depressive symptoms, 239 (14.5\%) exhibited moderate depressive symptoms, and 72 (4.4\%) might have moderately severe and more serious depressive symptoms. In terms of social support, close to onethird $(\mathrm{n}=457,27.7 \%)$ reported having no one to help with trivial things, the average number of people they could turn to for help was 0.98 ( $\mathrm{SD}=0.87)$; over one-third $(\mathrm{n}=569,34.4 \%)$ reported having no one to talk to when they felt down, and the average number of people they can talk to was also close to one (mean $=0.94, \mathrm{SD}=0.87$ ).

Correlational analyses were performed between UCLA 3-item, PHQ-9, social support proxies, living arrangement and age, and the results (Spearman's or Pearson's correlation coefficients) are summarised in table 2. The Spearman's correlation of Chinese UCLA 3-item with PHQ-9 scores was $0.42(\mathrm{p}<0.01)$, suggesting a moderate positive association between loneliness and depression. Loneliness was negatively correlated with emotional support $(\rho=-0.13)$, instrumental support $(\rho=-0.09)$, and age $(r=-0.05)$, and positively with living alone $(\rho=0.17)$; the correlations were all significant $(p<0.01)$ but weak. 
Table 2 Correlations between UCLA 3-item loneliness scale and selected variables

\begin{tabular}{|c|c|c|}
\hline Variable & Coefficients & $P$ value \\
\hline $\begin{array}{l}\text { 1. Scale item 1: lack companionship } \\
\text { ( } 0 \text { to } 3)\end{array}$ & $0.90^{*}$ & $<0.001$ \\
\hline 2. Scale item 2: feel left out (0 to 3 ) & $0.88^{*}$ & $<0.001$ \\
\hline $\begin{array}{l}\text { 3. Scale item 3: feel isolated from } \\
\text { others ( } 0 \text { to } 3 \text { ) }\end{array}$ & $0.89^{*}$ & $<0.001$ \\
\hline 4. PHQ-9 score (0 to 27$)$ & $0.42^{*}$ & $<0.001$ \\
\hline $\begin{array}{l}\text { 5. Emotional support ( } 0 \text { to } 5 \text { ) (No. of } \\
\text { people to talk to when feeling down) }\end{array}$ & $-0.13 \dagger$ & $<0.001$ \\
\hline $\begin{array}{l}\text { 6. Instrumental support ( } 0 \text { to } 5 \text { ) (No. } \\
\text { of people to help with everyday } \\
\text { tasks) }\end{array}$ & $-0.09 \dagger$ & $<0.001$ \\
\hline $\begin{array}{l}\text { 7. Living arrangement ( } 0 \text { to } 1 \text { ) (Living } \\
\text { with someone versus living alone) }\end{array}$ & $0.17 \dagger$ & $<0.001$ \\
\hline 8. Age & $-0.05^{\star}$ & $<0.001$ \\
\hline
\end{tabular}

*Pearson's correlation coefficients show individual item correlations with the 3-item loneliness scale.

†Spearman's correlation coefficients show the associations between the 3-item loneliness scale and related variables. PHQ-9, Patient Health Questionnaire-9.

Loneliness did not correlate with education or work years, and the results were not included in table 2. To further examine the incremental validity of loneliness in predicting depressive symptoms net of other risk factors, we performed hierarchical multiple regressions (table 3). Our final model (Model 3) explained $19 \%$ of the variance in PHQ-9 scores $(\mathrm{F}(6,1646)=67.44, \mathrm{p}<0.001)$, with loneliness explaining $18 \%$ unique variance, and age and emotional support remaining significant predictors.

\section{Optimal cut-off score of loneliness scale for detecting depressive symptoms}

Three ROC curve analyses were performed using PHQ-9 scores of 5, 10 and 15 as cut-offs, respectively, to detect mild, moderate and moderately severe depressive symptoms using loneliness scores. Applying this method, we calculated the optimal cut-offs with highest $\mathrm{J}$ for mild, moderate and moderately severe and more serious depressive symptoms. The results are summarised in table 4 and ROC curves shown in figure 1. To detect mild and more serious depressive symptoms, the optimal cut-off of loneliness score was 3 (sensitivity $76 \%$, specificity $62 \%$, positive predictive value (PPV) $84 \%$, negative predictive value (NPV) $51 \%$ ), the area under the ROC curve (AUC; SD) was $0.73 \pm 0.01$ (figure $1 \mathrm{~A}$ ) and significant $(z=17.17, \mathrm{p}<0.001)$. To detect moderate and more serious depressive symptoms, a cut-off of 4 was identified (sensitivity 69\%, specificity 55\%, PPV 26\%, NPV 88\%), the AUC (SD) was $0.66 \pm 0.02$ (figure $1 \mathrm{~B}$ ) and significant $(\mathrm{z}=9.01, \mathrm{p}<0.001)$. The same cut-off of 4 was chosen by $\mathrm{J}$ in detecting moderately severe and more serious depression symptoms (sensitivity 82\%, specificity 52\%, PPV $7.2 \%$, NPV 98.4\%), the AUC (SD) was $0.73 \pm 0.03$ (figure 1C) and significant as well $(\mathrm{z}=7.45, \mathrm{p}<0.001)$. A UCLA 3-item cut-off of 3 showed satisfactory sensitivity and specificity in detecting mild depressive symptoms among community-dwelling older adults, and a cut-off of 4 showed high sensitivity but low specificity in detecting moderately severe depressive symptoms.

\section{DISCUSSION}

We tested the use of a culturally adapt UCLA 3-item Loneliness Scale for detecting probable depression in a large Asian older sample. Our findings provided evidence that loneliness can be used as a construct for early detection of probable depression in community-dwelling older adults, with loneliness being a more acceptable and less stigmatising way for them to express depressive symptoms. This Chinese version of the UCLA 3-item scale in this study has good face and cultural validity, and internal consistency. Loneliness explained significant and unique variances of depressive symptoms after controlling for other risk factors, including social support and living arrangement,

Table 3 Regression models on PHQ-9 scores with different predictors

\begin{tabular}{|c|c|c|c|c|c|c|c|c|c|}
\hline \multirow[b]{2}{*}{ Variable } & \multicolumn{3}{|l|}{ Model 1} & \multicolumn{3}{|l|}{ Model 2} & \multicolumn{3}{|l|}{ Model 3} \\
\hline & B & SE(B) & $\boldsymbol{\beta}$ & B & SE(B) & $\boldsymbol{\beta}$ & B & SE(B) & $\boldsymbol{\beta}$ \\
\hline Constant & $10.44^{\star \star \star}$ & 1.00 & & $9.96^{\star \star \star}$ & 1.01 & & $6.27^{\star \star \star}$ & 0.93 & \\
\hline Female (ref: male) & 0.30 & 0.24 & 0.03 & 0.22 & 0.25 & 0.02 & 0.13 & 0.22 & 0.01 \\
\hline Living alone (0 to 1$)$ & -0.36 & 0.21 & -0.04 & -0.30 & 0.21 & -0.04 & $-0.91^{\star \star \star}$ & 0.19 & $-0.11^{\star \star \star}$ \\
\hline Instrumental support (0 to 5 ) & & & & -0.07 & 0.16 & -0.02 & -0.07 & 0.15 & -0.02 \\
\hline UCLA 3-item score (0 to 9) & & & & & & & $0.59^{\star \star \star}$ & 0.03 & $0.43^{\star * *}$ \\
\hline $\mathrm{R}^{2}$ & & $0.01^{* *}$ & & & $0.02^{*}$ & & & $0.19^{\star * \star}$ & \\
\hline$F(d f)$ & $7.95(3 t$ & 1649) & & $6.26(5$ & (1647) & & $67.44(6$ & 1646) & \\
\hline$\Delta \mathrm{R}^{2}$ & & $0.01^{* *}$ & & & $0.004^{*}$ & & & $0.18^{* *}$ & \\
\hline
\end{tabular}

${ }^{* * *} p<.000 ;{ }^{* *} p<0.01 ;{ }^{*} p<0.05$.

PHQ-9, Patient Health Questionnaire-9. 
Table 4 Sensitivity, specificity, AUC, PPV, NPV with 95\% Cl for each optional cut-off based on Youden's criterion

\section{No depression versus}

\section{$\quad$ moderate depressive $\quad$ moderately severe depressive}

$\geq$ mild depressive symptoms symptoms

symptoms

\begin{tabular}{llll}
\hline Cut-off & $\geq 3$ & $\geq 4$ & $\geq 4$ \\
\hline Sensitivity $(95 \% \mathrm{Cl})$ & $76.4(73.8$ to 78.8$)$ & $68.8(63.3$ to 73.9$)$ & $81.9(71.1$ to 90.0$)$ \\
\hline Specificity $(95 \% \mathrm{Cl})$ & $62.2(57.6$ to 66.6$)$ & $54.7(52.0$ to 57.4$)$ & $51.7(49.2$ to 54.2$)$ \\
\hline PPV $(95 \% \mathrm{Cl})$ & $83.6(80.9$ to 85.4$)$ & $26.0(24.0$ to 31.1$)$ & $7.2(6.5$ to 13.3$)$ \\
NPV $(95 \% \mathrm{Cl})$ & $51.0(47.6$ to 55.8$)$ & $88.3(85.6$ to 89.4$)$ & $98.4(97.2$ to 98.6$)$ \\
\hline J & 0.39 & 0.24 & 0.34 \\
AUC $(95 \% \mathrm{Cl})$ & $0.73(0.70$ to 0.75$)$ & $0.66(0.62$ to 0.69$)$ & $0.73(0.67$ to 0.79$)$ \\
\hline SE & 0.01 & 0.02 & $7.45^{* *}$ \\
\hline z & $17.17^{* * *}$ & $9.01^{* * *}$ & 0.03 \\
\hline
\end{tabular}

${ }^{* * *} \mathrm{p}<0.000 ;{ }^{* *} \mathrm{p}<0.01$

AUC, area under the curve; J, Youden's index; NPV, negative predictive value; PPV, positive predictive value.;

which revealed its incremental validity in its association with depression. The cut-off point of 3 on UCLA 3-item may signal mild depressive symptoms in communitydwelling older Chinese.

UCLA 3-item was designed and has been used in large scale surveys to detect loneliness; however, there are different ways of classifying 'lonely' about which there has been no consensus. For instance, some researchers classified 'lonely' as those who score in the top quintile of the total score, that is, 6 to $9,{ }^{50}$ while others classified those who answer 'some of the time' or 'often' to any item as lonely. ${ }^{31}$ Loneliness per se is a high risk factor for poor ageing outcomes and warrants attention; ${ }^{51}$ while the current findings further suggest its function to detect depressive symptoms. A cut-off point of 3 had the best balance between specificity and sensitivity to signal mild or more serious depressive symptoms in this sample of vulnerable and deprived community-dwelling older adults in an Asian community, and this cut-off point is lower than either the total quintile cut-off of $6^{50}$ or the item-by-item cut-off (minimun score 4) in detecting loneliness. ${ }^{51}$ It is possible that the lower cut-off found in this study is due to the expansion from three to four response categories, hence increasing the score range from 3 to 9 to 0 to 9 . It is also possible that in this already vulnerable and deprived population, of whom a high percentage are female, live alone, and are widowed/separated/ divorced/never married, a score of 3 on the UCLA 3-item may signify existing mild depressive symptoms.

The development and validation of the Chinese version of UCLA 3-item engaged professionals, front-line service providers and potential service users; and a standardised training protocol was also developed for training other people to use this screening tool. When designing a screening tool to be used in older adults population by mental health service providers in the community, many factors need to be considered, for example, suitable terminology for the population, older person's cognitive status, cultural issues, formal training for mental health professionals to execute the tools and referral pathway after administrating the tools. ${ }^{52}$ On a sensitive topic such as depression, a screening tool would work better if it can promote trust and rapport between the assessor and the older adult; and to develop such a tool, collaboration between service users and service providers is essential. There have been widespread calls to integrate service users and service providers in the development of mental health services in community-based settings; ${ }^{53}$ although
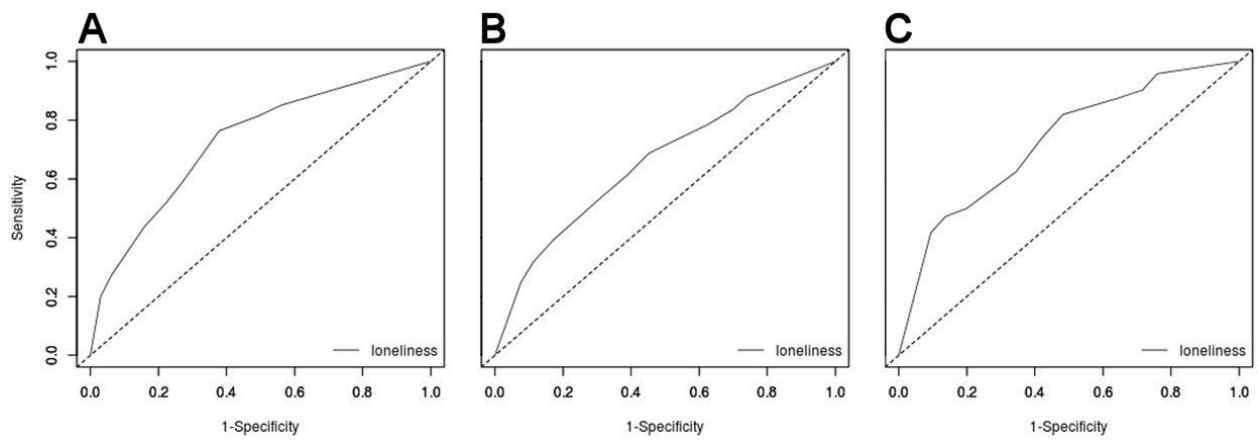

Figure 1 Receiver operating characteristic curve of the UCLA 3-item loneliness scale for the detection of (A) mild and more serious depressive symptoms (PHQ-9 $\geq 5)$, (B) moderate and more serious depressive symptoms (PHQ-9 $\geq 10)$, and (C) moderately severe and more serious depressive symptoms (PHQ-9 215). PHQ-9, Patient Health Questionnaire-9. 
the current study did not focus on engaging service users in service development per se, it embodied the concept of bridging the gap between research and service, and opened up windows to develop the partnership between researchers, service providers and service users in a meaningful manner.

With serious underdiagnosis of depression in older adults, methods to promote early detection is imperative. To achieve this, it is vital to detect mild depressive symptoms in the targeted population using easy-to-administer, culturally sensitive and validated assessment tools. This Chinese version of UCLA 3-item serves this purpose, and can easily be incorporated into a public education programme as a self-assessment tool to screen for depressive symptoms without mentioning the word depression, and a standardised protocol to use this scale is also in place and has been tested in training the social workers. In addition to detection of depressive symptoms in the community, loneliness is also associated with many other negative physical and mental health outcomes in older people, such as increased systolic blood pressure, ${ }^{51}$ increased anxiety and cognitive impairment. ${ }^{1254}$ Future studies could explore the nature of relationship and relevance of the UCLA 3-item as a quick screening tool for related health risks.

This study has several limitations. First, this sample of older adults was not randomly selected. Participants were recruited from community centres and through public education events among those who were suspected to be at risk of or with mild depressive symptoms. Although the profile of participants' PHQ-9 scores follows a normal distribution, this sample is more vulnerable than the normal population to other risk-prone demographic indicators. Therefore, whether data collected from the general population that includes mentally resilient older adults would shift the cut-off score remains unclear, and future studies are needed to affirm this using random sampling. Second, our measurement of social support provided information only on the number of available helping figures for emotional and instrumental support, and did not provide more detailed information about the frequency of help or contact. ${ }^{365}$ Third, this is a crosssectional study; despite the positive association between loneliness and depression, no causal relationship between loneliness and depression can be inferred with this data set. Future longitudinal data from this sample can provide further empirical evidence about the role of loneliness in predicting depression trajectory, which has implication values as when to intervene.

\section{CONCLUSION}

Loneliness is a unique risk factor for depressive symptoms in older adults, and Chinese older adults immersed in Asian Culture are more open to express and discuss about it than depression. Assessing loneliness may give us an invaluable opportunity for early detection and prevention of depression in old age. This study adapted the
UCLA 3-item loneliness scale for use in Chinese community, and this brief scale is well-suited as a screening tool for probably depression in the community. We have also developed and implemented the standardised protocol for training social workers to use the scale, and this protocol could be used for training other mental health service providers or community members who care about mental wellness of older people. This package of adapted loneliness scale, the training protocol and the cut-off scores can be used for task shifting and preventive care in mental health services.

Acknowledgements The authors would like to thank the project social workers for their help in recruiting participants and collecting data, research assistants for inputting and checking data, and all the participants for their contribution to this research.

Contributors TL and GW conceived the study and obtained funding. TL and WWK implemented the cultural adaptation of the UCLA 3-item scale. DKYL and LS coordinated scale adaptation, data input and data preparation. TL analysed and interpreted the data, wrote the first draft, which was refined in discussion with JYMT and HL for statistical analyses, and with SL for interpretation. GHKW and SL revised the draft further for intellectual consent. All authors read and approved the final manuscript.

Funding This work is supported by the Hong Kong Jockey Club Charites Trust for The University of Hong Kong for the Project JC JoyAge: Jockey Club Holistic Support Project for Elderly Mental Wellness (HKU Project Code: AR160026).

Competing interests None declared.

Patient consent for publication Not required.

Ethics approval This study was approved by the Human Research Ethics Committee (HREC) of the University of Hong Kong (HREC's reference number: EA1709021). Each participant provided written informed consent before participating in the study.

Provenance and peer review Not commissioned; externally peer-reviewed.

Data availability statement № data are available. The ethical approval and participant consent for this study do not allow sharing of data beyond the research team.

Supplemental material This content has been supplied by the author(s). It has not been vetted by BMJ Publishing Group Limited (BMJ) and may not have been peer-reviewed. Any opinions or recommendations discussed are solely those of the author(s) and are not endorsed by BMJ. BMJ disclaims all liability and responsibility arising from any reliance placed on the content. Where the content includes any translated material, BMJ does not warrant the accuracy and reliability of the translations (including but not limited to local regulations, clinical guidelines, terminology, drug names and drug dosages), and is not responsible for any error and/or omissions arising from translation and adaptation or otherwise.

Open access This is an open access article distributed in accordance with the Creative Commons Attribution Non Commercial (CC BY-NC 4.0) license, which permits others to distribute, remix, adapt, build upon this work non-commercially, and license their derivative works on different terms, provided the original work is properly cited, appropriate credit is given, any changes made indicated, and the use is non-commercial. See: http://creativecommons.org/licenses/by-nc/4.0/.

\section{ORCID iDs}

Tianyin Liu http://orcid.org/0000-0002-8194-5342

Hao Luo http://orcid.org/0000-0003-4261-3414

Gloria H Y Wong http://orcid.org/0000-0002-1331-942X

\section{REFERENCES}

1 Weeks DJ. A review of loneliness concepts, with particular reference to old age. Int J Geriatr Psychiatry 1994;9:345-55.

2 Hawkley LC, Cacioppo JT. Loneliness matters: a theoretical and empirical review of consequences and mechanisms. Ann Behav Med 2010;40:218-27. 
3 Gierveld JdeJ, de Jong Gierveld J. A review of loneliness: concept and definitions, determinants and consequences. Rev Clin Gerontol 1998;8:73-80.

4 Victor C, Scambler S, Bond J, et al. Being alone in later life: loneliness, social isolation and living alone. Rev Clin Geronto 2000;10:407-17.

5 de Jong Gierveld J, Tesch-Römer C. Loneliness in old age in eastern and Western European societies: theoretical perspectives. Eur $J$ Ageing 2012;9:285-95.

6 Yang $\mathrm{K}$, Victor $\mathrm{CR}$. The prevalence of and risk factors for loneliness among older people in China. Ageing Soc 2008;28:305-27.

7 Chen Y, Hicks A, While AE. Loneliness and social support of older people in China: a systematic literature review. Health Soc Care Community 2014;22:113-23.

8 Chou K-L, Chi I. Comparison between elderly Chinese living alone and those living with others. J Gerontol Soc Work 2000;33:51-66

9 Lou VWQ, Ng JW. Chinese older adults' resilience to the loneliness of living alone: a qualitative study. Aging Ment Health 2012;16:1039-46.

10 S-CJ Y, SK L. Living alone, social support, and feeling lonely among the elderly. Soc Behav Personal 2004;32:129-38.

11 Luo Y, Waite LJ. Loneliness and mortality among older adults in China. J Gerontol B: Psychol Sci Soc Sci 2014;69:633-45

12 Cacioppo JT, Hughes ME, Waite LJ, et al. Loneliness as a specific risk factor for depressive symptoms: cross-sectional and longitudinal analyses. Psychol Aging 2006;21:140.

13 Rodda J, Walker Z, Carter J. Depression in older adults. BMJ 2011;343:d5219.

14 van Zoonen K, Buntrock C, Ebert DD, et al. Preventing the onset of major depressive disorder: a meta-analytic review of psychological interventions. Int J Epidemiol 2014;43:318-29.

15 Djernes JK. Prevalence and predictors of depression in populations of elderly: a review. Acta Psychiatr Scand 2006;113:372-87.

16 Wetherell JL, Petkus AJ, McChesney K, et al. Older adults are less accurate than younger adults at identifying symptoms of anxiety and depression. J Nerv Ment Dis 2009;197:623.

$17 \mathrm{Ng} \mathrm{CH}$. The stigma of mental illness in Asian cultures. Aust N Z J Psychiatry 1997;31:382-90.

18 Lauber C, Rössler W. Stigma towards people with mental illness in developing countries in Asia. Int Rev Psychiatry 2007;19:157-78.

19 Chen R, Copeland JR, Wei L. A meta-analysis of epidemiological studies in depression of older people in the people's Republic of China. Int J Geriatr Psychiatry 1999;14:821-30.

20 Parker G, Gladstone G, Chee KT. Depression in the planet's largest ethnic group: the Chinese. Am J Psychiatry 2001;158:857-64.

21 Barg FK, Huss-Ashmore R, Wittink MN, et al. A mixed-methods approach to understanding loneliness and depression in older adults. J Gerontol B Psychol Sci Soc Sci 2006;61:S329-39.

22 Multi-function Chinese character database Hong Kong. Available: https://humanum.arts.cuhk.edu.hk/ [Accessed 28 Oct 2020].

23 Luanaigh CO, Lawlor BA. Loneliness and the health of older people. Int J Geriatr Psychiatry 2008;23:1213-21.

24 Heikkinen R-L, Kauppinen M. Mental well-being: a 16-year followup among older residents in Jyväskylä. Arch Gerontol Geriatr 2011;52:33-9.

25 Eloranta S, Arve S, Isoaho $\mathrm{H}$, et al. Loneliness of older people aged 70: a comparison of two Finnish cohorts born 20 years apart. Arch Gerontol Geriatr 2015;61:254-60.

26 Rubenowitz E, Waern M, Wilhelmson K, et al. Life events and psychosocial factors in elderly suicides-a case-control study. Psychol Med 2001;31:1193-202.

27 Russell D, Peplau LA, Cutrona CE. The revised UCLA loneliness scale: concurrent and discriminant validity evidence. J Pers Soc Psychol 1980;39:472-80.

28 Gierveld JDJ, Tilburg TV. A 6-item scale for overall, emotional, and social loneliness: confirmatory tests on survey data. Research on Aging 2006;28:582-98.

29 de Jong-Gierveld J. Developing and testing a model of loneliness. $J$ Pers Soc Psychol 1987;53:119.

30 Hughes ME, Waite LJ, Hawkley LC, et al. A short scale for measuring loneliness in large surveys: results from two population-based studies. Res Aging 2004;26:655-72.
31 Perissinotto CM, Stijacic Cenzer I, Covinsky KE. Loneliness in older persons: a predictor of functional decline and death. Arch Intern Med 2012;172:1078-84

32 Luo Y, Hawkley LC, Waite LJ, et al. Loneliness, health, and mortality in old age: a national longitudinal study. Soc Sci Med 2012;74:907-14

33 Shiovitz-Ezra S, Ayalon L. Use of direct versus indirect approaches to measure loneliness in later life. Res Aging 2012;34:572-91.

34 Pikhartova J, Bowling A, Victor $\mathrm{C}$. Is loneliness in later life a selffulfilling prophecy? Aging Ment Health 2016;20:543-9.

35 Beaton DE, Bombardier C, Guillemin F, et al. Guidelines for the process of cross-cultural adaptation of self-report measures. Spine 2000;25:3186-91.

36 Saito T, Cable N, Aida J, et al. Validation study on a Japanese version of the three-item UCLA loneliness scale among community-dwelling older adults. Geriatr Gerontol Int 2019;19:1068.

37 Chou K-L, Jun LW, Chi I. Assessing Chinese older adults' suicida ideation: Chinese version of the geriatric suicide ideation scale. Aging Ment Health 2005;9:167-71.

38 Wu Z-Q, Sun L, Sun Y-H, et al. Correlation between loneliness and social relationship among empty nest elderly in Anhui rural area, China. Aging Ment Health 2010;14:108-12.

39 Wang G, Hu M, Xiao S-Y, et al. Loneliness and depression among rural empty-nest elderly adults in Liuyang, China: a cross-sectional study. BMJ Open 2017;7:e016091.

40 Kroenke K, Spitzer RL, Williams JB. The PHQ-9: validity of a brief depression severity measure. J Gen Intern Med 2001;16:606-13.

41 Chi I, Chou KL. Social support and depression among elderly Chinese people in Hong Kong. Int J Aging Hum Dev 2001;52:231-52.

42 Lum TYS, Lou VWQ, Chen Y, et al. Neighborhood support and agingin-place preference among low-income elderly Chinese city-dwellers. J Gerontol B Psychol Sci Soc Sci 2016;71:98-105.

43 Chen Y-Y, Wong GH, Lum TY, et al. Neighborhood support network, perceived proximity to community facilities and depressive symptoms among low socioeconomic status Chinese elders. Aging \&Mental Health 2016;20:423-31.

44 Wong A, Nyenhuis D, Black SE, et al. Montreal cognitive assessment 5-minute protocol is a brief, valid, reliable, and feasible cognitive screen for telephone administration. Stroke 2015;46:1059-64.

45 Chemerinski E, Petracca G, Sabe L, et al. The specificity of depressive symptoms in patients with Alzheimer's disease. Am J Psychiatry 2001;158:68-72.

46 Starkstein SE, Mizrahi R, Power BD. Depression in Alzheimer's disease: phenomenology, clinical correlates and treatment. Int Rev Psychiatry 2008;20:382-8.

47 Youden WJ. Index for rating diagnostic tests. Cancer 1950;3:32-5.

48 Rosseel Y. Lavaan: an R package for structural equation modeling and more. version 0.5-12 (beta). J Stat Software 2012;48:1-36.

49 Hair JF, Anderson RE, Babin BJ, et al. Multivariate data analysis: a global perspective. Vol. 7. Upper Saddle River, NJ: Pearson, 2010.

50 Steptoe A, Shankar A, Demakakos P, et al. Social isolation, loneliness, and all-cause mortality in older men and women. Proc Natl Acad Sci U S A 2013;110:5797-801.

51 Hawkley LC, Thisted RA, Masi CM, et al. Loneliness predicts increased blood pressure: 5-year cross-lagged analyses in middleaged and older adults. Psychol Aging 2010;25:132-41.

52 Brijnath B, Gahan L, Gaffy E, et al. "Build rapport, otherwise no screening tools in the world are going to help": frontline service providers' views on current screening tools for elder abuse. Gerontologist 2020;60:472-82.

53 World Health Organization. Mental health action plan 2013-2020, 2013.

54 Fratiglioni L, Wang $\mathrm{H}-\mathrm{X}$, Ericsson $\mathrm{K}$, et al. Influence of social network on occurrence of dementia: a community-based longitudinal study. The Lancet 2000;355:1315-9.

55 Cairney J, Boyle M, Offord DR, et al. Stress, social support and depression in single and married mothers. Soc Psychiatry Psychiatr Epidemiol 2003;38:442-9. 